\title{
Analogía comparativa entre las redes sociales chinas y occidentales para la comercialización de productos servicios de alojamiento
}

DOI: https://doi.org/10.33262/ap.v2i1.25

(c) (i) ()

Comparative analogy between Chinese and Western social networks for the marketing of hosting services products

Diana Llanes Rosales ${ }^{1}$

\begin{abstract}
.
Tourism has undergone continuous expansion and diversification, becoming one of the economic sectors with the greatest growth in the world, including in this time of current crisis (García, 2013). One of the main points of the growth of tourists has been the arrival of the internet, because through this tool it is easier to carry out trips.

Keywords: Tourism, expansion, diversification, tools.

\section{Resumen.}

El turismo ha experimentado una continua expansión y diversificación, convirtiéndose en uno de los sectores económicos con mayor pujanza a nivel mundial, incluido en este tiempo de crisis actual (García, 2013). Uno de los principales puntos del crecimiento de turistas ha sido la llegada de internet, debido que a través de esta herramienta se hace más fácil la realización de los viajes.
\end{abstract}

Palabras claves: Turismo, expansión, diversificación, herramientas.

\footnotetext{
${ }^{1}$ Universidad de La Habana. Facultad de Turismo. La Habana, Cuba. dianallanes1995@gmail.com
} 


\section{Introducción.}

Internet se ha convertido en la plataforma que almacena un cúmulo de conocimiento, derivada de la gran cantidad de investigaciones e innovaciones elaboradas por el talento, la imaginación, la audacia y la inteligencia de los usuarios de la Red. Al mismo tiempo las redes sociales se han convertido en todo un fenómeno de masas, como en su momento lo constituyó el mundo de la blogosfera (Vivar, 2009). El nuevo turista se presenta cada vez más exigente. $\mathrm{Su}$ creciente experiencia en la compra y consumo de servicios turísticos, su mayor nivel cultural, la variedad de ofertas comerciales de la que goza y la mayor facilidad para buscar y obtener información -gracias a la aplicación de las nuevas tecnologías al sector-, hacen que el cliente exija productos cada vez más especializados, con la mejor relación calidad-precio. Al mismo tiempo son más activos y participativos en la búsqueda de información turística, en la preparación del viaje y en el consumo de los servicios adquiridos (Sánchez, 2015).

Los organismos públicos tienen interés en difundir el destino y las organizaciones privadas involucradas en la cadena productiva de turismo como hoteles, operadoras turísticas, agencias de viaje, transportadores, restaurantes, entre otros, buscan promover sus productos y servicios.

Por su parte, China es uno de los países más interconectados. El número de internautas en China llegó a 772 millones a finales del año 2018, de los cuales, 40.74 millones fueron sumados en 2017, un 5,6 por ciento más interanual, según un informe sobre el desarrollo de internet en China.

El Centro de Información de la Red de Internet de China (CNNIC) afirma que en junio de 2019 el 48.9\% de internautas chinos usan aplicaciones de reservas de viajes en línea (incluye boletos de avión, hoteles, tren y productos de viajes y vacaciones), alcanzando 418.15 millones.

China "continúa encabezando el turismo emisor mundial", según un informe publicado por la Organización Mundial de Turismo (OMT) de las Naciones Unidas. De acuerdo con el informe "Panorama OMT del turismo internacional", China sigue siendo el país que más gasta en el mundo, con una quinta parte del gasto en turismo internacional, seguida de los Estados Unidos, cifrados en 277 miles de millones en USD en el año 2018. Es por ello que los destinos a nivel internacional se han enfocado en China como destino emisor, siendo atractivo para la mayoría de los destinos de América Latina y el Caribe, panorama donde Cuba muestra gran interés en este mercado potencial.

Aun cuando China no se encuentra entre los primeros mercados emisores de la isla, de acuerdo a datos de la ONEI (Oficina nacional de Estadísticas de Cuba) del año 2018 pues las primeras tres posiciones son ocupadas por Canadá (903 275 visitantes), la comunidad cubana (491 992 visitantes) y los Estados Unidos (521 846 visitantes) se conoce que a partir del año 2010, la tasa promedio anual de crecimiento de las llegadas de turistas chinos a Cuba ha sido 
del $23 \%$. En el 2018 se reportaron cifras significativas de viajeros chinos (alrededor de 49 000 visitantes), con un $10 \%$ del total general de los excursionistas, cifra que se prevé vaya en ascenso, pues en el año 2017, la cifra de visitantes chinos sólo ascendía a 43322 viajeros. Razón por la cual China se ubica como un importante mercado emisor de turistas no solo para el orbe y países de Latinoamérica, igualmente va ocupando un lugar importante en el destino-país Cuba.

En el contexto de la cultura china a la hora del uso de los canales de información, el turista chino prioriza para su selección aquellos destinos y establecimientos de alojamiento que tengan un buen "Kou bei" (en español, "comentarios"). Tomando en consideración los comentarios que realizan sus compatriotas para planificar y seleccionar sus viajes al extranjero. Prestando especial atención a la calidad y las comodidades de los hoteles de los destinos que visitan. De ahí que la presencia en estos sitios de búsqueda definidos, para este segmento en específico, resulte de vital importancia para insertarse de forma estratégica en él.

\section{"Las redes sociales como fortalecedores de la demanda: su relacion con los servicios de alojamiento."}

Las redes sociales son la evolución de las tradicionales maneras de comunicación del ser humano, que ha avanzado con el uso de nuevos canales y herramientas, y que se basan en la creación, conocimiento colectivo y confianza generalizada (Fonseca, 2014)

Las principales características de la llamada "red social" son: concepto de comunidad, a través de la creación de redes de usuarios que interactúan, dialogan y aportan comunicación y conocimiento; tecnología flexible y ancho de banda necesario para el intercambio de información y estándares web de aplicación libre; y una arquitectura modular que favorece la creación de aplicaciones complejas de forma más rápida, a un menor coste. Mientras en el comercio electrónico 1.0 los usuarios eran meros receptores de servicios, en el comercio electrónico 2.0 producen contenidos (blogosfera), participan del valor (intercambio) y colaboran en el desarrollo de la tecnología. El proceso de comunicación genera, en definitiva, un flujo activo de participación (Freire, 2008).

Las redes sociales al ser un medio de carácter social mediante el cual se puede compartir estados de ánimo e información personal sobre intereses ya sea en forma de fotos, videos, etc; posee una fuerte influencia en el proceso de inspiración y planificación de los viajes.

En resumen, las redes sociales más allá de lo que exprese su definición semántica, en la actualidad es considerada por todos como una herramienta útil, creadora de un espacio de interacción social que permite el surgimiento, acercamiento y consolidación de relaciones entre uno o más individuos. 
En lo que se refiere a la promoción de destinos de forma concreta, la OMT señaló en 1999 que los “destinos triunfadores serán los que, por medio de Internet, puedan satisfacer la red de información de los turistas y puedan persuadirlos de que ese lugar merece el tiempo y el dinero que se gastarán en visitarlos (Castellucci, 2007).

Las redes sociales se convierten en generadoras de la imagen del destino, construida por la mente del turista (Méndez, 2015).

La presencia de las empresas en las redes sociales les permite integrarse en ambientes en red de conversación, al tiempo que ocupan los espacios que correspondan a la denominación de la marca y de sus productos. Por su parte, los medios proyectan su identidad de marca y ofrecen servicios (como titulares, encuestas y concursos) en las redes sociales abiertas y dan soporte a redes sociales propias como estrategia de fidelización e interactividad con los usuarios.

Los hoteles por su parte, también están aprovechando las tecnologías para diseñar sus estrategias de mercadeo porque internet mejora la accesibilidad, comodidad, rapidez, novedad, cobertura global, riqueza de la información, flexibilidad y coste reducido que permite a los huéspedes conocer los servicios desde cualquier lugar del mundo y desde cualquier dispositivo (Morales, Agüera, \& Cuadra, 2015).

El sitio de opinión más importante del sector turístico y hotelero es TripAdvisor con más de 830 millones de opiniones sobre más de 8.6 millones de alojamientos, compañías aéreas, atracciones y restaurantes. Alberga la comunidad de viajeros más grande del mundo con 460 millones de visitantes únicos al mes. Se encuentra disponible en 49 mercados y 28 idiomas ("Información sobre Tripadvisor," 2020).

La reputación online en el sector hotelero es muy importante debido a que los productos ofrecidos son intangibles y no pueden ser evaluados antes de su consumo, incrementando la importancia de los comentarios de otras personas. Muchos productos turísticos y hoteleros se perciben como compras de alto riesgo, por lo que las opiniones del grupo son un aspecto importante en el proceso de decisión de compra (Diana-Jens \& Rodríguez, 2015).

Resumiendo, las redes sociales son un canal de marketing digital para el comercio electrónico, indispensable en los servicios de alojamientos, pues el uso eficiente de las redes sociales permite atraer nuevos clientes, incrementar las ventas hoteleras, reforzar la reputación online y con ello la imagen de la organización. Además de servir como puente en las relaciones con los huéspedes, así como crea una fuente de contenido sobre los clientes, esencial en la toma de decisiones.

\section{"el usuario chino versus el usuario occidental":}

La presencia de internautas chinos aumenta en la red. Son cada vez más numerosos y más participativos, generando debate y crítica en la sociedad digital a través de los llamados 
microblogs locales, ya que las redes sociales extranjeras, como Twitter o Facebook, se encuentran, censuradas por el gobierno (Tudela, 2011).

Según el Centro de Información de la Red de Internet de China (CNNIC), a junio de 2019, China tenía 854.49 millones de internautas, hasta 25,98 millones desde finales de 2018, y su penetración en Internet alcanzó el 61,2\%, 1,6 puntos porcentuales más que a fines de 2018.

A partir de junio de 2019, el número de reservas de viajes en línea usuarios en China habían alcanzado 418.15 millones, 8,14 millones más que a finales de 2018, lo que representa el $48,9 \%$ de todos los usuarios de Internet.

La distribución en China está muy fragmentada y se desarrolla íntegramente de forma online, a través de los marketplaces, los principales son: Apple Store (28\%), Tencent Myapp (14\%), 360 Mobile Assistant (10,4\%), Baidu Mobile Assistant (9\%), Oppo App Store (9\%), Huawei App Market (8,2\%), MIUI App Store (6,2\%), Vivo App Store (5,2\%), PP Asistant (2\%), Wandoujia $(1,4 \%)$ y otros $(6,6 \%)$. Asimismo, en referencia a la comunicación, se emplea un modelo multicanal que combina herramientas tanto offline como online (Pastor, 2018).

El principal canal de comunicación continúa siendo las redes sociales liderando con un 53\%. Por otro lado las sugerencias de amigos o familiares, los sitios web oficiales y los anuncios online son canales que disfrutan de un nivel de popularidad casi igualado.

Dentro del interminable mundo digital, resulta inevitable el desarrollo que han alcanzado algunas redes sociales en el campo del turismo hacia el mercado occidental. Entre ellas se encuentran:

1. Facebook, Instagram y Youtube como las plataformas favoritas de los departamentos de marketing de las empresas turísticas y oficinas de turismo cuando se trata de contratar campañas de publicidad a través de redes sociales.

2. Facebook e Instagram son los canales más efectivos tanto para construir marcas como para obtener una respuesta directa entre los consumidores.

3. Facebook es la consulta de búsqueda más popular en todo el mundo. Existen más de 70 millones de empresas que poseen una página de Facebook. Más de 20 millones de esos utilizan Messenger para comunicarse con sus clientes.

4. Instagram es uno de los mayores gigantes de redes sociales en el mercado en este momento con más de mil millones de usuarios activos mensuales y más de 25 millones de cuentas comerciales activas. El 80\% de los Instagrammers siguen un negocio en Instagram.

5. Alrededor del $80 \%$ de los influencers consideran a Instagram como una forma principal de colaboración con las marcas. 
6. El $60 \%$ de las personas afirman que descubren nuevos productos en Instagram.

Es un hecho que los viajeros chinos están más inclinados a usar canales digitales (aplicaciones móviles, foros en línea y sitios web, entre otros) para obtener información y hacer reservas para alojamiento y actividades. Sin embargo, debido a las restricciones en la República Popular China de servicios muy famosos en occidente (Facebook, Instagram, etc.), las empresas de servicios chinas han desarrollado sus propias versiones (mercados, redes sociales, mapas, correo electrónico, etc.) adaptadas a los gustos locales. Estas aplicaciones pioneras han creado un abanico de servicios que han superado con creces a sus análogos occidentales.

A continuación, se presenta un listado de las principales redes sociales usadas por este segmento (sin un orden particular):

- Dazhong Dianping (大众点评): esta aplicación es parecida a Tripadvisor. Se trata de una plataforma de intercambio de opiniones e información sobre restaurantes, karaokes, bares, cines, actividades, negocios locales y otros lugares de ocio tanto dentro como fuera de China.

- Ctrip (携程旅游): Esta empresa shanghainesa es la mayor web de servicios de viaje del país, sus homólogas occidentales son Expedia y Booking.com. Reserva de billetes de avión, autobús o trenes, reserva de hoteles y apartamentos (más de 110000), cruceros, alquiler de transporte y guías turísticos privados (algunos con servicios en inglés y español). También ofrece paquetes de viaje individualizados, compra de entradas para espectáculos, lugares turísticos y museos, salidas y viajes organizados, guías turísticas para leer online totalmente gratuitas, compras de souvenirs y comida típica, planes para el fin de semana, excursiones, etc. Otras aplicaciones móviles muy parecidas en idioma chino son Qunar (去哪儿), Tuniu (途牛旅游) y Mafengwo (马 蜂窝).

- Tujia (途家): Es una agencia de viajes de servicio completo con sede en Beijing, China, que apunta al mercado de viajes chino. Esta plataforma ha sido llamada "Airbnb de China", ya que su modelo es muy similar al suyo: los usuarios de Tujia pueden publicar listados de propiedades que pueden alquilarse. Sin embargo, a diferencia de Airbnb, no permite que los propietarios individuales publiquen listados de sus casas, para anunciar sus propiedades deben pasar primero por los agentes y gerentes de propiedades. Xiaozhu (小猪) es al igual que Tujia una aplicación inmobiliaria.

- QQ: es un servicio de mensajería instantánea parecido a Messenger pero con muchas más funciones: la nube, noticias, videojuegos, compras, videollamadas, chat de voz, o crear y unirse a grupos de cualquier temática. Este chat es el más antiguo del país 
(fue lanzado en 1999), por lo que es una herramienta muy útil para comunicarnos con amigos o empresas tanto dentro como fuera de China.

- Wechat (微 信): es una aplicación que reúne las funciones de diversas redes occidentales como Skype, Whatsapp, Tindr, Google Wallet, Facebook e eBay.Cada vez es más común que empresas o autónomos vendan servicios y productos a través de Wechat, o pagar en hoteles, bares o máquinas expendedoras con esta aplicación.

- QZone (空间): esta aplicación es parecida a Facebook. Permite la creación de un perfil (por lo general asociado a una cuenta de QQ) y subir tu propia información personal, actualizar tu blog o diario, crear álbumes de fotos, hacer nuevos amigos, escuchar música o ver videos.

- Sina Weibo (新浪微博): esta aplicación de móvil es un espacio para microblogs parecida a Twitter. Alrededor de 100 millones de mensajes cortos se publican cada día en esta app.

De las diez redes sociales más usadas en todo el mundo, cinco provienen de China, lo que evidencia el nivel de aceptación que poseen las mismas. Liderando la lista se encuentran las principales redes occidentales como Facebook, Youtube, WhatsApp y por supuesto Messenger, una extensión de Facebook. Sin embargo, la red oriental Wechat según sus cifras supera al bien conocido Instagram, lo que refleja su incersion en el mercado occidental, estando presente en el 86\% de los dispositivos. Por detrás le siguen QQ, Qzone y Sina weibo con cifras superiores incluso a redes como Twitter, Snapchat.

"Posicionamiento de los servicios de alojamiento del destino la habana en las plataformas online":

Figura 1. Posicionamiento de los servicios de alojamiento del destino la habana en las plataformas online

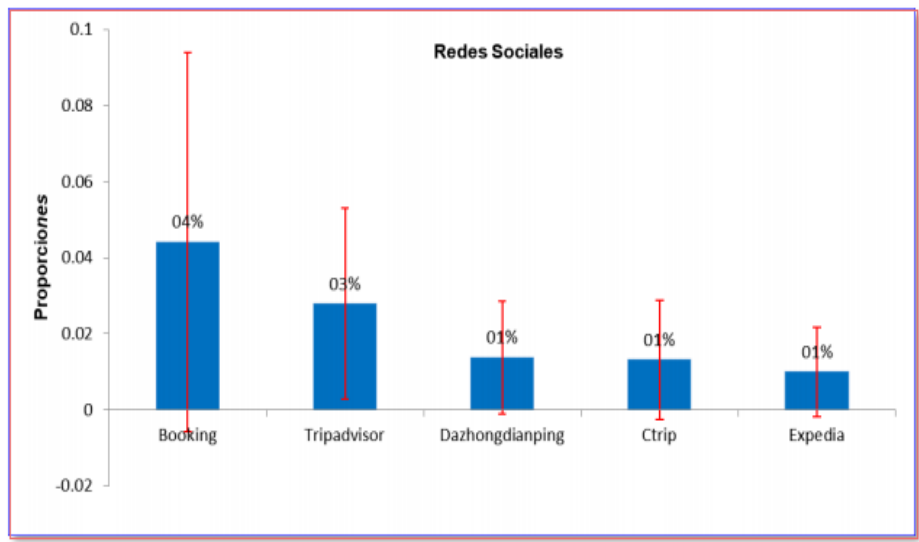

Fuente: Elaboración propia. 
Diferentes plataformas digitales marcan la presencia de las instalaciones hoteleras de La Habana. Entre ellas se encuentran los sitios web: Ctrip, Dazhongdianping, Expedia, Booking y Tripadvisor. Muchas de ellas se conectan entre sí, redirigiendo al usuario de un sitio a otro, en especial las tres últimas. La figura 1 demuestra que Tripadvisor y Booking son los sitios de mayor presencia de alojamientos. Mientras que las redes chinas como Ctrip y Dazhongdianping no cuentan con la misma cantidad de establecimientos, representando sólo el $0.1 \%$ del total de alojamientos.

La red social más conocida y usada a la hora de valorar instalaciones hoteleras, especialmente hoteles es Tripadvisor. Dentro de los hoteles más comentados por dicha red occidental se encuentran: Iberostar Parque Central, Hotel Nacional, Habana Libre, Meliá Habana Hotel, Sevilla, Saratoga, y por último, aunque en menor medida el hotel Santa Isabel. La mayoría de las opiniones sobre estos hoteles giran en torno a comentarios sobre la calidad de los servicios, de las instalaciones en especial del mobiliario, la actitud de los trabajadores, etc.

Figura 2. Comentarios

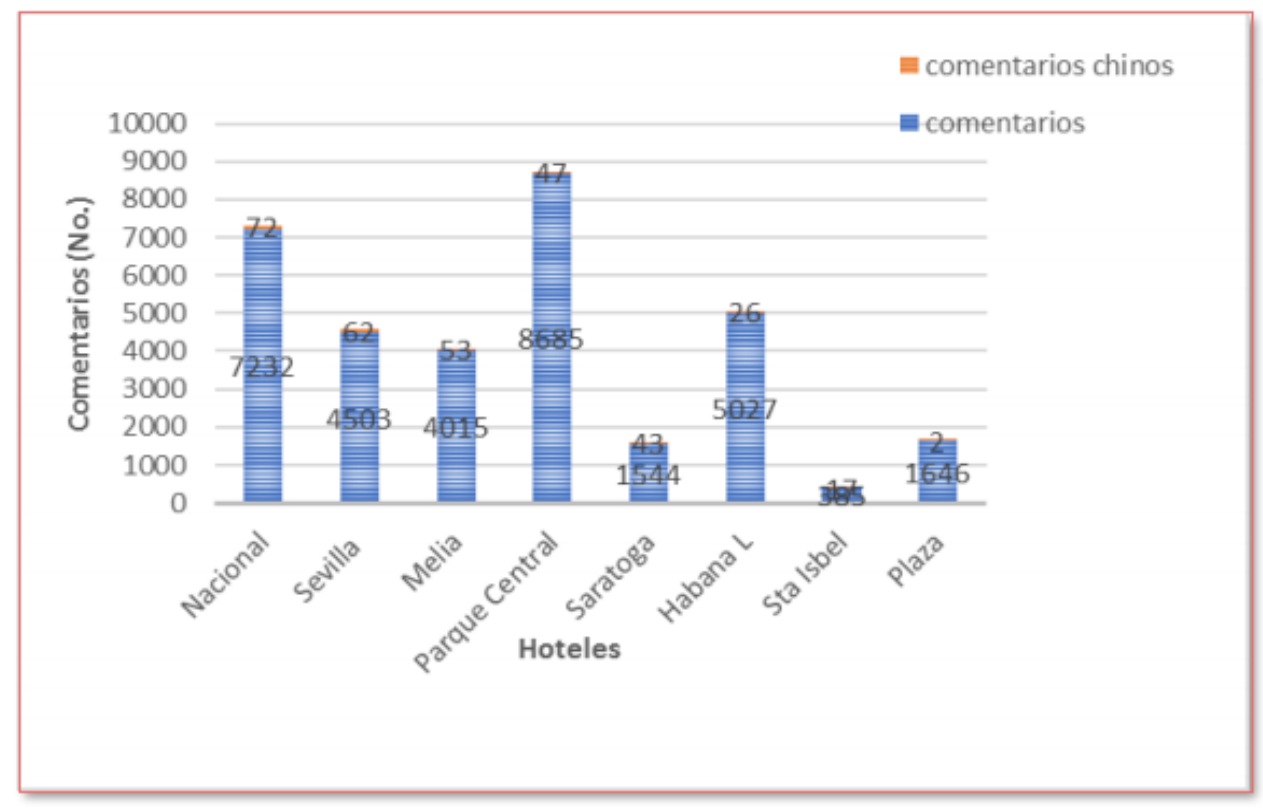

Fuente: Elaboración propia.

Los hoteles Nacional, Sevilla, Meliá Habana como se muestra en la figura 2 son los que poseen mayor cantidad de comentarios chinos, pues son hoteles bien posicionados por sus valores históricos y culturales. Hoteles como El Parque Central y Habana Libre a pesar de que gozan de la aceptación de otros mercados teniendo gran número de comentarios, no posee mucha aceptación por parte de los turistas chinos. Marcas como Iberostar y Kempinski representadas en los hoteles Gran Packard y Gran Manzana Kempinski no gozan de mucha popularidad en esta red social, 'pues no tienen presencia de comentarios chinos. 
Sin embargo, la figura 3 refleja que el hotel Santa Isabel junto al Saratoga son los que tienen mayor porciento de comentarios chinos, con un 4,2\% y $2,7 \%$ respectivamente, lo que demuestra que son los preferidos para este mercado por su confort, accesibilidad de precios y ubicación.

Dazhongdianping, red analoga de Tripadvisor, reporta como los hoteles más comentados son el Hotel Manzana Kempinski (20 comentarios), seguido del Hotel Nacional (17 comentarios). En comparación a Tripadvisor todos los comentarios de esta red pertenecen únicamente a turistas chinos., pero su porcentaje es casi mínimo, lo que refleja que no existe un posicionamiento en dicha red, a pesar de contar con disímiles multimedia ya sea videos cortos y/o fotos de lugares turísticos de la Habana como hoteles, restaurantes, lugares históricos (El Morro de la Cabaña), etc.

Figura 3. Comentarios chinos

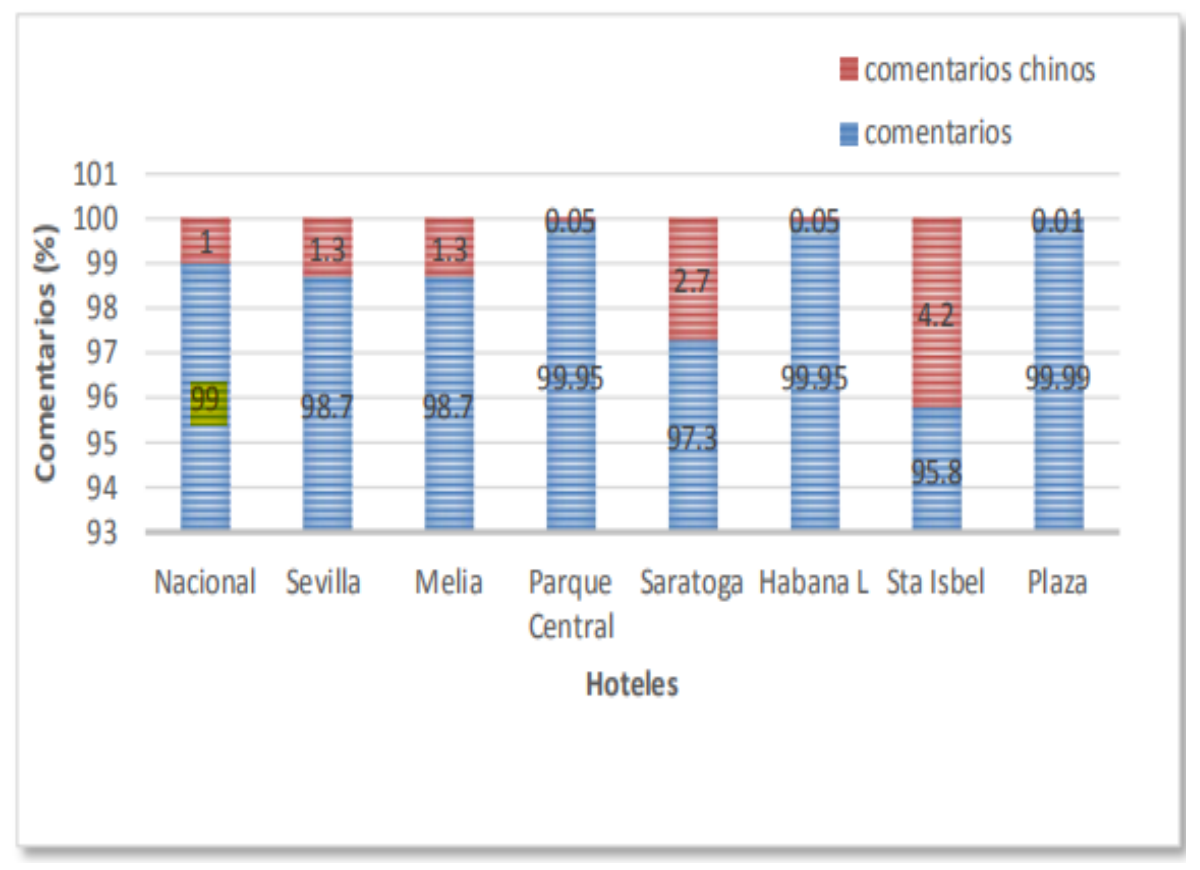

Fuente: Elaboración propia.

Expedia y Boking.com son dos de las principales plataformas utilizadas en el proceso de reserva, estas venden fundamentalmente a grupos de viajeros a través de agencias de viajes. A pesar de que es utilizada por turistas chinos a la hora de reservar alojamiento en destinos occidentales, en dichas redes casi no existen comentarios de turistas chinos. Los mismos son principalmente de turistas de origen canadiense y estadounidense.

Ctrip por su parte, como refleja la figura 4, tiene varios hoteles preferidos por los turistas chinos. Resalta por la gran cantidad de comentarios el Hotel Nacional donde casi la totalidad de cometarios pertenecen al mercado chino. 
Figura 4. Hoteles preferidos

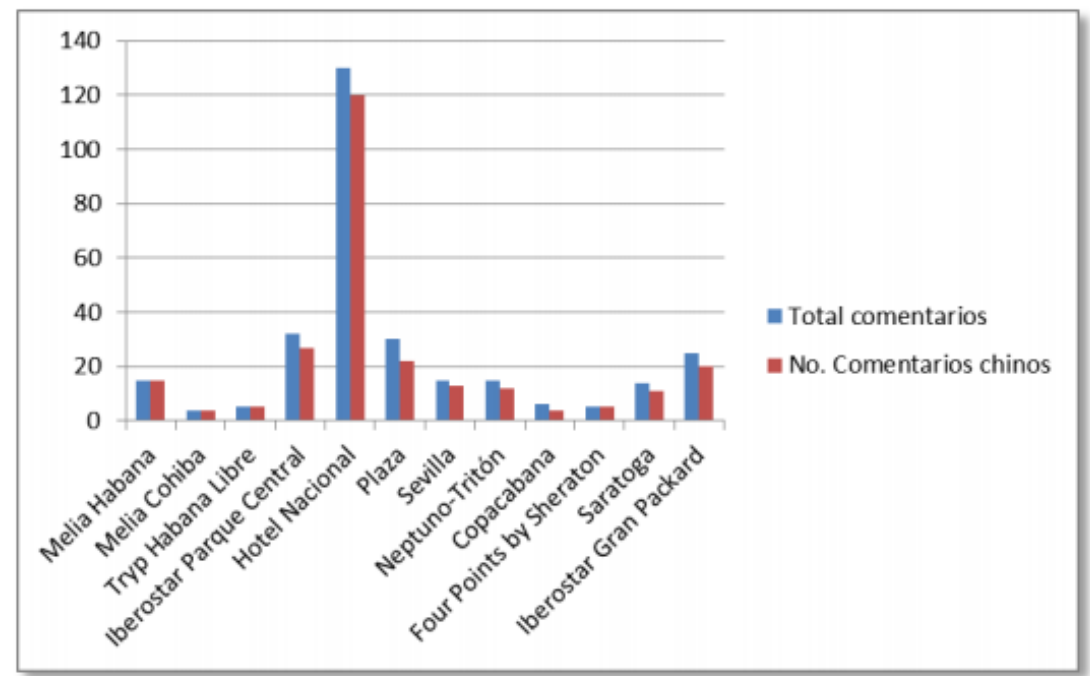

Fuente: Elaboración propia.

Los resultados demuestran que la comercialización en plataformas chinas como Ctrip y Dazhongdianping, no es suficiente, pues presentan los índices más bajos de comentarios, al contrario de otras plataformas como Tripadvisor.

Por otra parte, como resultado del proceso de apertura al turismo, muchas familias abrieron sus casas para hospedar a turistas de diferentes regiones. Como resultado el servicio de alojamiento en la habana además de contar con la participación de los hoteles, una gran parte descansa en las casas de renta. Es por ello que la plataforma Airbnb cuenta con el mayor número de propiedades, teniendo más 600 propiedades localizadas en el destino La Habana de alrededor de 1000 casas en toda Cuba.

En comparación con Airbnb, Tujia es una empresa inmobiliaria que a pesar de que posee más de 11000 casas de renta disponibles en más de 150 destinos alrededor de todo el mundo, ubicándose el mayor número de casas en territorio chino, sin embargo solo hay presencia de no más de 10 casas particulares de la Habana.

\section{Conclusiones.}

- Concluimos entonces que el mercado chino es muy exigente cuando se trata de las condiciones de alojamiento, esto se condiciona al trato del personal, la buena comunicación basada en el dominio del idioma chino e inglés, la accesibilidad de precios, entre otros pero sin lugar a dudas las redes sociales poseen una influencia indirecta tanto en la realización del primer viaje al destino como al principal motivo del viaje, lo que refleja la insuficiente información turística de La Habana en redes chinas. 
- Las agencias de viajes juegan, por tanto, un papel importante en relación con la primera visita, así como la cantidad de veces que se visite el destino. La presencia de páginas online gubernamentales con información sobre el destino La Habana, la relación con agencias de viajes importantes chinas que cuenten con sus propias redes sociales y apps, y por último la existencia de la página web de la embajada china con informaciones turísticas constituyen fortalezas esenciales para el aprovechamiento de las oportunidades. Fundamentalmente en lo que concierne a la promoción de los productos y/o servicios mediante la diversificación de los mecanismos de promoción y comercialización a partir de los soportes a emplear en función de los nuevos escenarios de comunicación digital (internet, aplicaciones, entre otras), asi como el empleo de las redes sociales en especial las chinas, así como de promoción en el idioma chino y la inserción de la información del destino en los principales buscadores chinos. Porque si bien los chinos consideran el turismo como un elemento fundamental de su filosofía de vida, el acceso a este segmento requiere de una comprensión más estrecha y de canales que distan un poco de los convencionales para el mercado occidental.

\section{Referencias bibliográficas.}

Andrade, A. V. B., Varela, M. C. G., \& Vega, C. P. G. (2016). Comercio electrónico: Imprenta Universitaria Buhalis, D., \& Jun, S. H. (2011). E-Tourism.

Castellucci, D. (2007). Aplicación de las TICs en la promoción de destinos. Aportes y transferencias, 1 .

Celaya, J. (2008). La Empresa en la WEB 2.0. España: Editorial Grupo Planeta.

Colliva, J. L. O. (2008). Internet: la hora de las redes sociales.

Conde, E., Schmidt, N., \& Ochoa, I. (2011). El Turismo Eletrónico,una necesidad para las empresas del sector. TURyDES, No.4.

Cross-Border Data Flows Enable Growth in All Industries. (2015): Information Technology and Innovation Foundation

Diana-Jens, P., \& Rodríguez, A. (2015). La reputación online y su impacto en la política de precios de los hoteles. Cuadernos de Turismo, 36, 129-155.

Digital Globalization: The New Era of Global Flows. (2016): McKinsey Global Institute. .

Fadraga, A. H. (2020). Diseño de una ruta turística de compras para el mercado emisor chino en el destino La Habana. . Universidad de La Habana.

Fan, J. (2015). The Alibaba Effect: Spatial Consumption Inequality and the Welfare gains from e-commerce. Shanghai University of Finance and Economics?Michigan State University. 
Fesenmaier, W. P. (2012). The Role of Smartphones in Mediating the Touristic Experience. Journal of Travel Research.

Fonseca, A. (2014). Marketing Digital en Redes Sociales: Lo imprescindible en Marketing Online para tue empresa en las Redes Sociales: Alexandre Fonseca Lacomba.

Freire, F. C. (2008). Las redes sociales trastocan los modelos de los medios de comunicación tradicionales. Revista Latina de Comunicación Social, 11.

García, F. G. (2013). Agencias de viaje online de España: aplicación de un modelo de análisis de sedes web. TURyDES, revista de investigación en turismo y desarrollo local, 6.

Gariboldi, G. (1999). Comercio electrónico: conceptos y reflexiones básicas (Vol. 4): BIDINTAL.

Gretzel, U. (2018). Tourism and Social Media. Sage Handbook of Tourism Management.

Higuerey, E. (2019). Comercio electrónico: conoce todo sobre este modelo de negocios y cuáles son sus ventajas. . Retrieved from http:/rockcontent.com/es/blog/comercioelectronico/

Hosteltur. (2013). Internet y turismo: el campo de batalla cinco años después del boom 2.0. Hosteltur.

Hosteltur. (2019). Redes sociales preferidas por el turismo para el marketing digital Hosteltur.

Información sobre Tripadvisor. (2020). from www.tripadvisor.mediaroom.com

Kandampully, J., Keating, B. W., Kim, B., Mattila, A. S., \& Solnet, D. (2014). Service research in the hospitality literature: insights from a systematic review. Cornell Hospitality Quarterly, 55(3), 287-299.

Méndez, N. R. (2015). La promoción turística a través de las redes sociales: el caso de los ayuntamientos gallegos de menos de 5.000 habitantes. Universidad Da Coruña.

Montalvo, S., \& Andrada, M. (2005). Investigación comercial y de mercados. Andalucía.

Morales, Agüera, \& Cuadra. (2015). Análisis de las variables que influyen en la reputación online de las empresas turísticas. el caso de los hoteles de córdoba y granada. Revista De Investigaciones Turísticas, 11, 103-120

Nodarse, F. F. (2013). Sobre Comercio electrónico en la WEB 2.0 y 3.0. Revista Cubana de Ciencias Informáticas.

Oropeza, D. K. (2018). La competencia económica en el comercio electrónico y su protección en el sistema jurídico mexicano México: UNIVERSIDAD NACIONAL AUTÓNOMA DE MÉXICO. INSTITUTO DE INVESTIGACIONES JURÍDICAS.

Pan, B. (2015). E-Tourism. Parkin, M. (2010). Economics. 
Pastor, J. A. (2018). Economía colaborativa en el sector del turismo en China ICEX ( España, exportación e innovaciones).

Pérez, M. P., Sanchéz, A. M., \& Garcés, S. A. (2014). El Sector Turístico y el Comercio Electrónico. Profile of Chinese Outbound Tourists: Characteristics and Expenditures. (2014). American Journal of Tourism Management.

Rosales, D. L. (2017). China y el Turismo en América Latina y Cuba. Su integración en los bloques regionales.

Sambhanthan, A., \& Good, A. (2012). Strategic Advantage in Web Tourism Promotion: An e-Commerce Strategy for Developing Countries

Sampieri, R. H., Collado, C. F., \& Lucio, M. d. P. B. (2010). Metodología de la investigación.

Sánchez, R. C. (Facultad de Ciencias de la Comunicación. Universidad de Málaga). EL ARTE DE COMUNICARSE CON EL NUEVO TURISTA. De las nuevas necesidades turísticas y las nuevas estrategias de comunicación empresarial e institucional.

Siaw, L. P., Ansah, G. O., \& Adjei, D. (2015). E-Commerce: An Opportunity For Hospitality Industry Growth. Case Study Of Hotels In Accra Researchjournali's Journal of Hospitality Tourism.

Sikander, V. S. y. J. (2010). A perspective architecture for electronic commerce and digital marketing. Microsoft Corporation-Mindtree.

Thomaz, G. M., Biz, A. A., \& Gândara, J. M. (2013). Innovación en la promoción turística en medios y redes sociales: Un estudio comparativo entre destinos turísticos. Estudios y perspectivas en turismo, 22(1), 102-119.

Tudela, R. Á. (2011). Las redes sociales en China bajo censura. Ábaco, Vol. 2. UNWTO. (2019). Asian Tourism Trends UNWTON, \& Academy, C. T. (2019). Guidelines for Success in the Chinese Outbound Tourism Market.

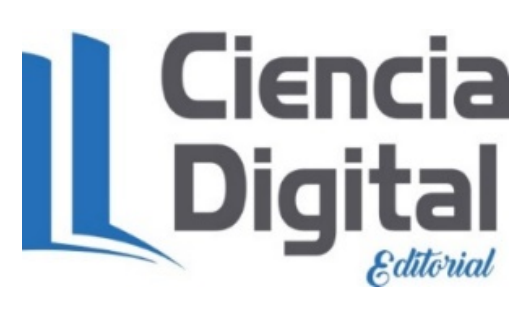




\section{Para citar el artículo indexado}

Llanes Rosales, D. (2020). Analogía comparativa entre las redes sociales chinas y occidentales para la comercialización de productos servicios de alojamiento. AlfaPublicaciones, 2(1), 6-19. https://doi.org/10.33262/ap.v2i1.25

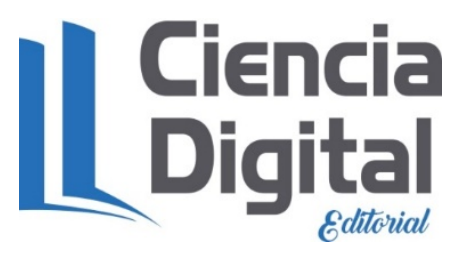

El artículo que se publica es de exclusiva responsabilidad de los autores y no necesariamente reflejan el pensamiento de la Revista Alpha Publicaciones.

El artículo queda en propiedad de la revista y, por tanto, su publicación parcial y/o total en otro medio tiene que ser autorizado por el director de la Revista Alpha Publicaciones.
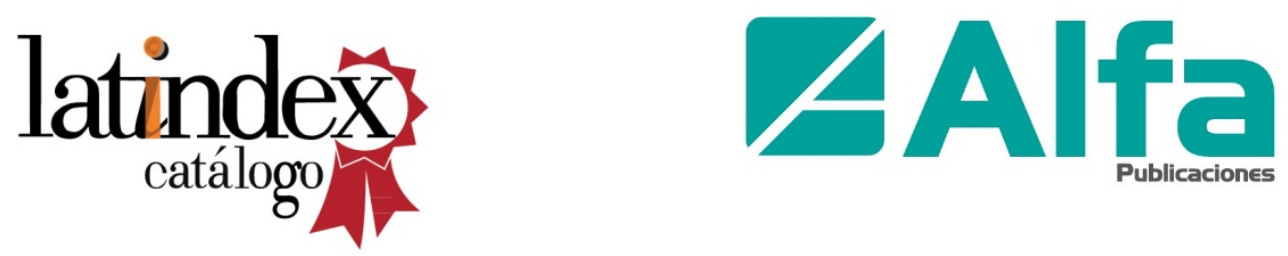